\title{
SCIENTIFIC REP RTS \\ The fastest animals and vehicles are neither the biggest nor the fastest over lifetime
}

Received: 19 February 2018

Accepted: 27 July 2018

Published online: 27 August 2018

\author{
A. Bejan $\mathbb{1}^{1}$, U. Gunes $\mathbb{D}^{1,3}$, J. D. Charles ${ }^{2}$ \& B. Sahin ${ }^{3}$
}

Here we show how the size of a body affects its maximum average speed of movement through its environment. The theoretical challenge was to predict that 'outliers' must exist, such as the cheetah for terrestrial animals and the jet fighter for airplanes. We show that during a travel that starts from rest and continues at cruising speed, the body size for minimum travel time, or maximum average speed, is not the biggest. The results are compared with extensive data for military aircraft for chase, attack and reconnaissance, in addition to data for commercial aircraft. The paper also explains why in earlier studies of flying (animals, airplanes) the airplane data deviated upward (toward greater speeds) relative to the theoretical trend followed by flying animals, and why the fastest animal flyers are one thousand times smaller than the fastest swimmers. Unlike the biggest animals and airplanes (elephant, whale, commercial jet), which move constantly, the fastest animals and airplanes spend most of their lives at rest. When judged for speed averaged over lifetime, the fastest 'sprinters' are in fact the slowest movers (as in Aesop's fable 'The Tortoise and the Hare').

Bigger bodies tend to move faster on land, in water, and in the air. This broad trend is supported by the speed-size data collected from many sources for flying, running and swimming (Fig. 1) ${ }^{1}$. On this unifying background, outliers exist. The burst speed of the cheetah is greater than the steady speed of the elephant. The tuna can swim faster than the steady whale. There are outliers that are even more distant from the general trend, but they should not be confused with the fastest runners, swimmers and fliers. For example, there are insects (e.g., the flea) that snap and jump at speeds much greater than what their speed-mass scaling law would predict. The ant odontomachus holds this kind of speed record (of order $200 \mathrm{~km} / \mathrm{h}$ ) by snapping its jaws shut, in order to jump to the side, to get out of the way of danger. This is a one-shot event, not cyclical locomotion. Most of her life, this ant walks on land at ant speed, in accord with the speed-mass scaling law for animal locomotion ${ }^{1}$.

Hirt et al. ${ }^{2}$ addressed this aspect of animal locomotion with a model chosen to account for the starting (accelerating) period in the movement of the fastest animals. The model consisting of elastic fast-twitch fibers was constructed such that the speed emerges as a concave function of body size, with its peak at a body size that is not the largest size (Fig. 2). Bejan ${ }^{3}$ observed that the existence of body size for peak speed also rules the evolutionary design of jet fighter aircraft, which is the human made counterpart of the animal of prey, with a high burst speed followed by a long period of inactivity on the ground.

The following analysis is a theoretical treatment of this physics aspect of locomotion. It is a generalization of the unifying theory of animate, inanimate and vehicle movement, which includes the lifetime and life travel of animate and inanimate movement ${ }^{4}$.

Additionally, the following theory accounts for several features of the alignment of locomotion data that have not been questioned. One is the fact that the data for airplanes in Fig. 1 are not aligned with the data for animal fliers. Why are the airplane data trending above the animal speed-mass correlation? Why do the airplane data fall on a steeper line than the data for animal fliers? Moving over to Fig. 2, why does the body size for peak speed decrease in the direction from swimming to running and flying?

${ }^{1}$ Duke University, Department of Mechanical Engineering and Materials Science, Durham, NC, 27708-0300, USA. ${ }^{2}$ Blue Origin, 21218 76th Ave S, Kent, WA, 98032, USA. ${ }^{3}$ Yildiz Technical University, Department of Naval Architecture and Marine Engineering, Besiktas, Istanbul, 34349, Turkey. Correspondence and requests for materials should be addressed to A.B. (email: abejan@duke.edu) 


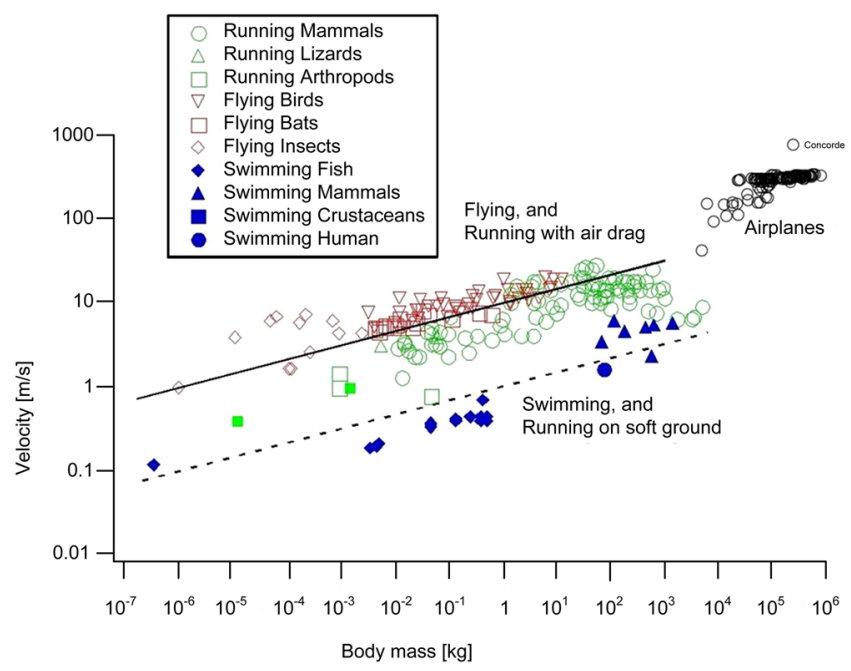

Figure 1. Animal speed-mass data (fliers, runners, swimmers) compiled from sources indicated in ref. ${ }^{1}$

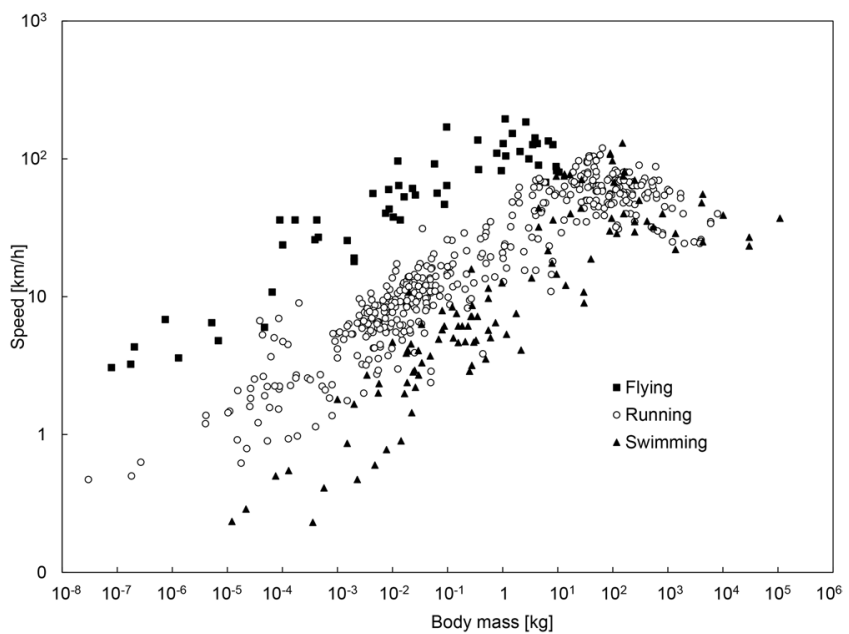

Figure 2. Animal speed-mass data compiled after Hirt et al. ${ }^{2}$, showing that the peak speed belongs to animals that are close to but not the biggest. The data are compiled from sources indicated in ref. ${ }^{2}$.

\section{Theory}

Consider the body of a vehicle or animal of size $\mathrm{M}[\mathrm{kg}]$ moving horizontally on the earth's surface to a distance L. The body starts from rest $(\mathrm{V}=0)$, is then accelerated to the cruising speed $\mathrm{V}_{c}$ over the distance $\mathrm{L}_{\mathrm{a}}$ and time interval $t_{a}$. The body continues its travel at cruising speed $V_{c}$ over the distance $L_{c}$ and time interval $t_{c}$. We ask what size (M) enables the body to travel the distance $\mathrm{L}=\mathrm{L}_{\mathrm{a}}+\mathrm{L}_{\mathrm{c}}$ the fastest.

The body $\mathrm{M}$ moves because it is driven by power. The power comes from an engine (animal, or human made). The power produced by the engine for the purpose of forcing the motion is ${ }^{4}$

$$
\dot{\mathrm{W}}=\eta \dot{\mathrm{Q}}
$$

where $\dot{\mathrm{Q}}=\dot{\mathrm{m}} H$ is the rate of heat transfer that drives the engine, $\dot{\mathrm{m}}[\mathrm{kg} / \mathrm{s}]$ is the rate of fuel (or food) consumption, and $\mathrm{H}[\mathrm{J} / \mathrm{kg}]$ is the heating value of the consumed fuel.

Recent physics articles ${ }^{5,6}$ showed that for maximum efficiency the engine size must have the same scale as the body size $M$, which is why in the following analysis $M$ is the scale of the body plus the engine. In other words, the moving body has a single size scale, $\mathrm{M}$. Next, the phenomenon of economies of scale requires the efficiency to increase monotonically with the body size,

$$
\eta=\mathrm{C}_{1} \mathrm{M}^{\alpha}
$$

where the exponent $\alpha$ is less than 1 , for example, $\alpha=1 / 4$ for helicopter engines ${ }^{7}$, and $\alpha=5 / 12$ for animal locomotion (flying, running, swimming) ${ }^{4}$. In Fig. 3 we show that economies of scale are also present in the evolution of jet engines for aircraft, and that the exponent $\alpha$ is of order 0.14 . The data ${ }^{8}$ plotted in Fig. 3 are tabulated in 


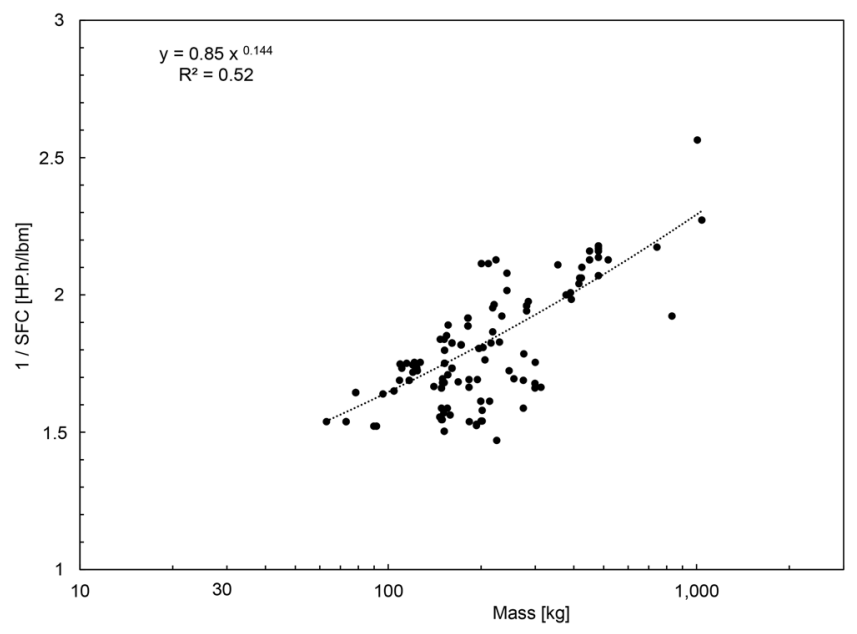

Figure 3. The effect of size on the efficiency of jet engines for aircraft. The data are tabulated in Supplementary Material.

Supplementary Material. The $\mathrm{R}^{2}$ value is 0.52 and the $\mathrm{P}$ value is below 0.001 , therefore the correlation is statistically significant ${ }^{9}$. Combining Eqs (1) and (2) we find that the power that drives the body is

$$
\dot{\mathrm{W}}=\mathrm{C}_{1} \mathrm{HM}^{\alpha} \dot{\mathrm{m}}
$$

The power is dissipated in three ways:

(i) To accelerate the body,

(ii) To lift the body (or an equivalent body of water in the case of swimmers ${ }^{1}$ ), and

(iii) To overcome the drag posed by the medium relative to which the body is moving.

We distinguish two sequential regimes of movement: acceleration, defined such that (ii) and (iii) are negligible compared with (i), and cruise, in which (i) is negligible compared with (ii) and (iii).

\section{Acceleration}

During acceleration, the power exerted by the motor on the body is matched by the force of accelerating the mass $(\mathrm{MdV} / \mathrm{dt})$ times the instantaneous speed $\mathrm{V}$,

$$
\mathrm{C}_{1} \mathrm{HM}^{\alpha} \dot{\mathrm{m}}=\mathrm{M} \frac{\mathrm{dV}}{\mathrm{dt}} \mathrm{V}
$$

Integrating this equation from $t=0$ to $t=t_{a}$, where $V=V_{c}$, we find

$$
\begin{aligned}
& \mathrm{m}_{\mathrm{a}}=\frac{\mathrm{M}^{1-\alpha} \mathrm{V}_{\mathrm{c}}^{2}}{2 \mathrm{C}_{1} \mathrm{H}} \\
& \mathrm{t}_{\mathrm{a}}=\frac{\mathrm{M}^{1-\alpha} \mathrm{V}_{\mathrm{c}}^{2}}{2 \mathrm{C}_{1} \mathrm{H} \overline{\dot{m}}_{\mathrm{a}}} \\
& \mathrm{L}_{\mathrm{a}}=\frac{\mathrm{M}^{1-\alpha} \mathrm{V}_{c}^{3}}{3 \mathrm{C}_{1} \mathrm{H} \overline{\dot{m}}_{\mathrm{a}}}
\end{aligned}
$$

where $m_{a}$ is the amount of fuel used over the acceleration period, and $\overline{\mathrm{m}}_{\mathrm{a}}$ is the average fuel consumption rate $\left(\mathrm{m}_{\mathrm{a}} / \mathrm{t}_{\mathrm{a}}\right)$ during that period.

The theoretical cruising speed $V_{c}$ emerged ${ }^{1}$ as the trade off between the power spent on lifting (ii) and the power spent on overcoming drag (iii). The cruising speed depends monotonically on body size,

$$
\mathrm{V}_{\mathrm{c}}=\mathrm{C}_{2} \mathrm{M}^{1 / 6}
$$

where $C_{2}=\operatorname{rg}^{1 / 2} \rho^{-1 / 6}$, where $\rho$ is the body density, and $\mathrm{r}$ accounts for the medium in which the body is moving: $\mathrm{r}=\left(\rho / \rho_{\mathrm{a}}\right)^{1 / 3}$, where $\rho$ is the body density and $\rho_{\mathrm{a}}$ is the density of the ambient. Representative ranges of values are $\mathrm{r} \sim 10$ for fliers, $\mathrm{r} \sim 1$ for swimmers, and $1<\mathrm{r}<10$ for terrestrial locomotion. Combined, Eqs (6) and (8) confirm one side of the trade off noted at the start: bigger bodies take longer to be accelerated to their cruising speed: 


$$
\mathrm{t}_{\mathrm{a}}=\frac{\mathrm{M}^{4 / 3-\alpha} \mathrm{C}_{2}^{2}}{2 \mathrm{C}_{1} \mathrm{H} \overline{\dot{\mathrm{m}}_{\mathrm{a}}}}
$$

\section{Cruise}

The corresponding analysis for the cruising period begins with Eq. (8), where $\mathrm{V}_{c}=\mathrm{L}_{c} / \mathrm{t}_{c}$. The engine work spent over $\mathrm{L}_{\mathrm{c}}$ is

$$
\mathrm{W}_{\mathrm{c}} \sim \mathrm{r}^{-1} \mathrm{Mg} \mathrm{L}_{\mathrm{c}}=\mathrm{C}_{1} \mathrm{HM}^{\alpha} \overline{\mathrm{m}}_{\mathrm{c}} \mathrm{t}_{\mathrm{c}}
$$

The travel time $t_{c}$ is shorter when the body is bigger, and this confirms the second side of the trade off,

$$
\mathrm{t}_{\mathrm{c}}=\frac{\mathrm{L}_{\mathrm{c}}}{\mathrm{C}_{2} \mathrm{M}^{1 / 6}}
$$

\section{The size trade off}

A first glimpse at the trade off that determines the body size for shortest travel time is possible if we assume that the fuel consumption rate is represented by a known constant, $\dot{\mathrm{m}}_{\mathrm{a}}=\dot{\mathrm{m}}_{\mathrm{c}}$. The analysis begins with Eq. (11) by replacing $L_{c}$ with $\left(L-L_{a}\right)$, where $L$ is fixed and $L_{a}$ is given by Eq. (7). The total travel time, or the inverse of the speed averaged over the distance $\mathrm{L}$, is

$$
\frac{\mathrm{t}_{\mathrm{a}}+\mathrm{t}_{\mathrm{c}}}{\mathrm{L}}=\frac{\mathrm{C}_{2}^{2}}{6 \mathrm{C}_{1} \mathrm{HL} \overline{\mathrm{m}}_{\mathrm{a}}} \mathrm{M}^{4 / 3-\alpha}+\frac{1}{\mathrm{C}_{2}} \mathrm{M}^{-1 / 6}
$$

with the condition that $\mathrm{L}_{\mathrm{a}}<\mathrm{L}$. Equation (12) can be nondimensionalized by introducing the cruising speed of a reference body of fixed unit mass, $\mathrm{M}_{0}=1 \mathrm{~kg}$, namely $\mathrm{V}_{0}=\mathrm{C}_{2} \mathrm{M}_{0}^{1 / 6}$. Equation (12) becomes

$$
\frac{1}{\widetilde{V}_{\text {avg }}}=\mathrm{AM} \widetilde{M}^{4 / 3-\alpha}+\widetilde{M}^{-1 / 6}
$$

where $\widetilde{\mathrm{V}}_{\mathrm{avg}}=\mathrm{V}_{\mathrm{avg}} / \mathrm{V}_{0}$ and $\mathrm{V}_{\mathrm{avg}}=\mathrm{L} /\left(\mathrm{t}_{\mathrm{a}}+\mathrm{t}_{\mathrm{c}}\right)$,

$$
\begin{gathered}
\frac{1}{\widetilde{V}_{\text {avg }}}=\frac{\mathrm{t}_{\mathrm{a}}+\mathrm{t}_{\mathrm{c}}}{\mathrm{L}} \cdot \mathrm{C}_{2} \mathrm{M}_{0}^{1 / 6} \\
\widetilde{\mathrm{M}}=\frac{\mathrm{M}}{\mathrm{M}_{0}} \\
\mathrm{~A}=\frac{\mathrm{C}_{2}^{3} \mathrm{M}_{0}^{3 / 2-\alpha}}{6 \mathrm{C}_{1} \mathrm{HL} \overline{\mathrm{m}}_{\mathrm{a}}}
\end{gathered}
$$

Equation (13) shows that the total travel time has a minimum with respect to body size. Alternatively, the speed averaged over the total travel has a maximum with respect to body size. The design for peak velocity is represented by

$$
\begin{gathered}
\widetilde{\mathrm{M}}_{\text {peak }}=\mathrm{A}^{\frac{-1}{3 / 2-\alpha}} \mathrm{B}_{\mathrm{M}}(\alpha) \\
\widetilde{\mathrm{V}}_{\text {peak }}=\mathrm{A}^{\frac{-1 / 6}{3 / 2-\alpha}} \mathrm{B}_{\mathrm{V}}(\alpha)
\end{gathered}
$$

where

$$
\begin{gathered}
\mathrm{B}_{\mathrm{M}}(\alpha)=\left[6\left(\frac{4}{3}-\alpha\right)\right]^{\frac{-1}{3 / 2-\alpha}} \\
\mathrm{B}_{\mathrm{V}}(\alpha)=\left(\mathrm{B}_{\mathrm{M}}^{4 / 3-\alpha}+\mathrm{B}_{\mathrm{M}}^{\frac{-1 / 6}{3 / 2-\alpha}}\right)^{-1}
\end{gathered}
$$

Figure 4 shows that the factors $\mathrm{B}_{\mathrm{M}}$ and $\mathrm{B}_{\mathrm{V}}$ are both of order 1 , and are relatively insensitive to changes in the exponent $\alpha$.

These results are in accord with the model of Hirt et al. ${ }^{2}$ : the largest animal is not necessarily the fastest. The fastest travel emerges at an intermediate size in animals (and vehicles) where the accelerating period is not negligible in comparison with the total travel time. This feature belongs to animals of prey and jet fighters. Another 


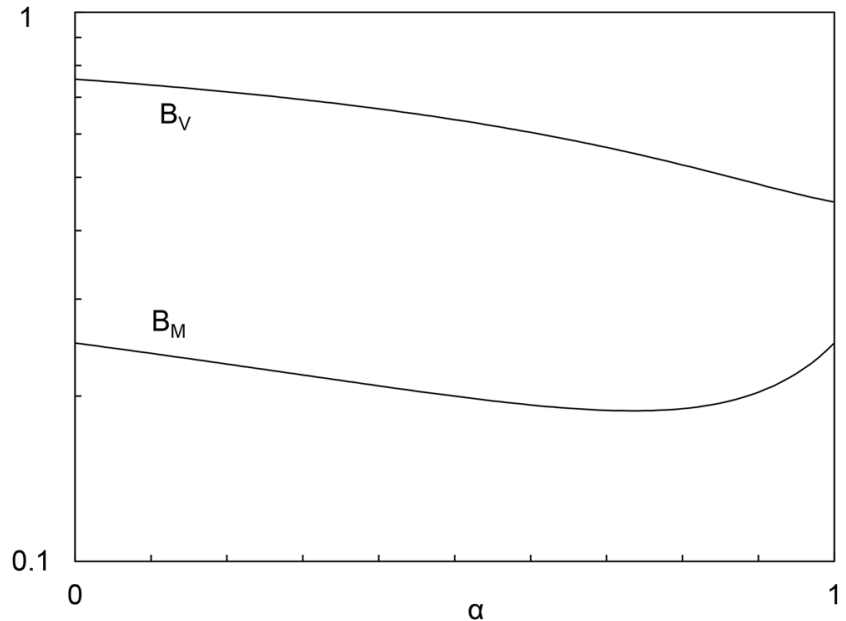

Figure 4. The insensitivity of factors $B_{M}$ and $B_{v}$ to changes in $\alpha$.

conclusion is that even the fastest [Eqs (17) and (18)] obey the theoretical ${ }^{1}$ proportionality between speed and mass raised to the power $1 / 6$, as in Eq. (8), namely

$$
\widetilde{V}_{\text {peak }}=\frac{B_{V}}{B_{M}^{1 / 6}} \widetilde{M}_{\text {peak }}^{1 / 6}
$$

The analysis presented above is based on the simplifying assumption that the fuel consumption rate $\dot{m}$ is a constant. If we also account for the body size effect on $\dot{m}$, we can repeat the analysis by replacing $\dot{m}$ with $\mathrm{C}_{3} \mathrm{M}^{\beta}$, where, as we show next, the exponent $\beta$ is approximately $1 / 2$. The $\beta$ exponent has been found previously in two ways, empirically for commercial airplanes ${ }^{6}$, and theoretically for animals ${ }^{4}$. Specifically, for airplanes the fuel load is roughly $M / 3$, the range is statistically proportional to $\mathrm{M}^{0.64}$, and if we take the average speed to be proportional to $\mathrm{M}^{1 / 6}$ we conclude that $\beta \cong 0.53$. For animals, the analysis detailed in ref. ${ }^{10}$ concluded with $\beta=0.5$. This means that in the results presented above $\mathrm{C}_{1}$ is replaced by $\mathrm{C}_{1} \mathrm{C}_{3}$, and $\alpha$ is replaced by $\alpha+\beta$. In other words, the value of the exponent $\alpha \lesssim 0.5$, which was used until now, is replaced by an exponent with a value $\alpha+\beta \lesssim 1$. Looking at Fig. 4 , we see that the change from 0.5 to 1 on the abscissa does not have a meaningful effect on $\mathrm{B}_{\mathrm{M}}, \mathrm{B}_{\mathrm{V}}$ and Eq. (21).

\section{The fastest airplanes}

The quest for speed in human flight has a rich history dominated by the development of military aircraft for attack and reconnaissance ${ }^{10}$. Highlights are collected chronologically in Fig. 5 (top) and Fig. 6. The data plotted in these figures are tabulated in Supplementary Material. The sequence of frames in Fig. 6 focuses progressively on earlier stages of the development of fast models. Figure 7 (top) shows the evolution of the speeds of military aircraft over time.

The broad view conveyed by Figs 5-7 is that during the past eight decades the fastest models have been joined by even faster models that are also bigger. The theoretical trend derived in Eq. (21) matches the cloud of data of commercial aircraft and early military models compiled in Fig. 5 (top). Several approximating assumptions were made in tracing the two lines, namely $\mathrm{r} \approx 10, \mathrm{~B}_{\mathrm{v}} \approx 1.5$ and $\mathrm{B}_{\mathrm{M}} \approx 0.2$. For the average density of the aircraft $[\rho$ in the $\mathrm{C}_{2}$ formula under Eq. (8)] we used the method of ref. ${ }^{11}$, which shows that the density of the fuselage of the B747400 is $31.9 \mathrm{~kg} \mathrm{~m}^{-3}$, or 3 percent of the density of water. We indicated this order of magnitude as the band between $\rho=\rho_{\text {water }} / 100$ and $\rho=\rho_{\text {water }} / 10$.

Most of the data for the fastest military models fall above the trend traced with Eq. (21) in Fig. 5 (top). The explanation has its origin in the fact that the early military models flew at subsonic speeds and at altitudes comparable with those of contemporary commercial models. Although the fastest military models since the 1950s have supersonic speeds, they also fly at higher altitudes.

Figure 7 (bottom) complements Fig. 5 (top) by showing the altitude ceiling of each of the models. At higher altitudes the air density is lower, and the $\mathrm{r}$ factor (in $\mathrm{C}_{2}$, and later in $\mathrm{V}_{\text {peak }}$ ) is greater. Specifically, if in the $\mathrm{r}$ definition

$$
r=\left(\frac{\rho}{\rho_{\mathrm{a}}}\right)^{1 / 3}
$$

$\rho_{\mathrm{a}}$ is the air density at low altitudes that are comparable with ground level, then the correct $\mathrm{r}$ factor that belongs in the theoretical result for $\mathrm{V}_{\text {peak }}$ [Eq. (21)] is

$$
r=\left(\frac{\rho}{\rho_{\mathrm{a}, \text { ceiling }}}\right)^{1 / 3}=\left(\frac{\rho}{\rho_{\mathrm{a}, \text { ground }}}\right)^{1 / 3} \mathrm{R}
$$



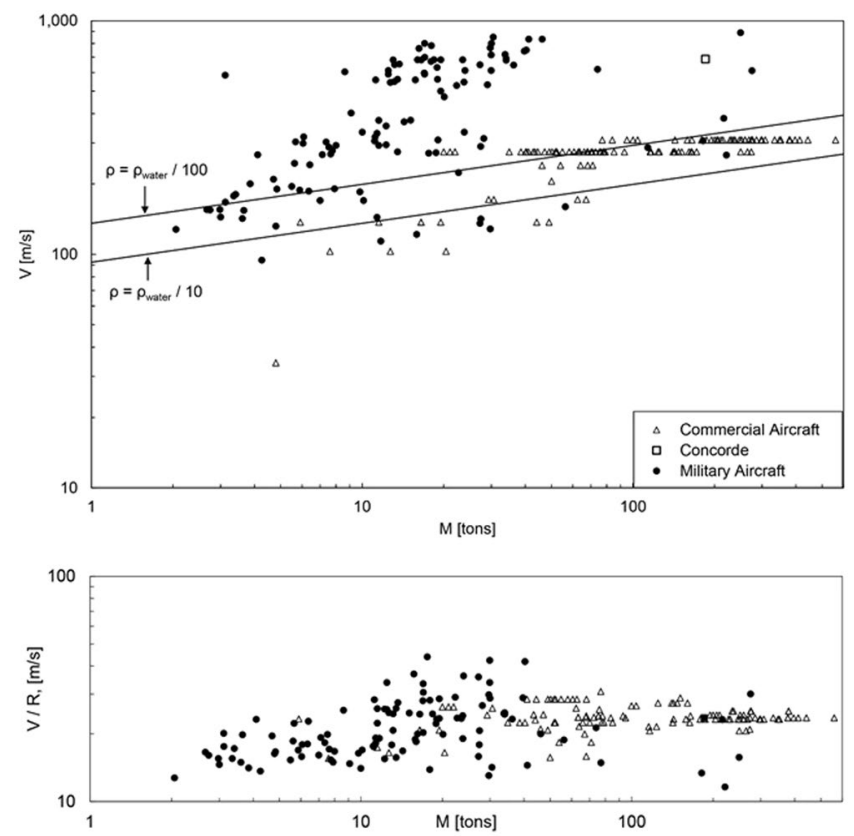

Figure 5. Speed versus size in military and commercial aircraft. Bottom: the military aircraft data collapse on the commercial aircraft data and the predicted theoretical trend when the effect of altitude is included.

where

$$
\mathrm{R}=\left(\frac{\rho_{\mathrm{a} \text {,ground }}}{\rho_{\mathrm{a} \text {,ceiling }}}\right)^{1 / 3}
$$

The new factor $\mathrm{R}$ is monotonically greater than 1 as the ceiling altitude plotted in Fig. 7 (bottom) increases.

\section{Why airplanes deviate from flying animals}

The flying altitude has a predictable effect on the maximum theoretical speed, Eq. (23), because $V_{\text {peak }}$ is proportional to $\mathrm{r}$. To bring this effect into view, in Fig. 5 (bottom) we repeated the data of Fig. 5 (top) by plotting on the ordinate $\mathrm{V} / \mathrm{R}$ in place of $\mathrm{V}$. This way we removed the effect of altitude, in other words, we referenced all the theoretical speeds to the $\mathrm{r}$ value that corresponds to air density at ground level.

The R values employed in Fig. 5 (bottom) come from Eq. (24) and the relationship between altitude and atmospheric air density ${ }^{12}$. For example, at an altitude of $10 \mathrm{~km}$ the air density is $0.41 \mathrm{~kg} / \mathrm{m}^{3}$ at $-50^{\circ} \mathrm{C}$, while at ground level at $20^{\circ} \mathrm{C}$ it is $1.2 \mathrm{~kg} / \mathrm{m}^{3}$. In this numerical example $\mathrm{R}$ is approximately 0.15 .

Compare Fig. 5 (bottom) with Fig. 5 (top). The speed data collapse on the theoretical trend obeyed by animals when the altitude effect $(\mathrm{R})$ is taken into account. The correctness of the theory is strengthened if we replot the classical animal speed data of Fig. 1 as $V / R$ versus $M$, in place of $V$ versus $M$. This new representation is provided in Fig. 8. It is now evident why in the original version of Fig. 1 the airplane data deviate above the theoretical trend. With the effect of altitude taken into account, the theory covers correctly all the speed data, from animals (Fig. 1) to commercial and military aircraft (Figs 5-7). Noteworthy is also that the airplane data condensed now in the V/R-revised Fig. 5 (bottom) and Fig. 8 show a hump (i.e., a maximum speed at an intermediate size), which is reminiscent of the animal data plotted in Fig. 2.

\section{Why the fastest fliers are significantly smaller than the fastest swimmers}

The animal data compiled in Fig. 2 show that the peak speeds of animals have the same order of magnitude $(100 \mathrm{~km} / \mathrm{h})$, but the fastest flier is three orders of magnitude smaller than the fastest swimmer. Here is why this effect is in accord with the theory that concluded with Eqs (17-20):

Note that the body size for peak speed $\left(M_{\text {peak }}\right)$ is proportional to A raised to the power $-1 /(3 / 2-\alpha)$, where $\alpha$ is positive and smaller than 1 . In other words, the theoretical $M_{\text {peak }}$ is expected to vary roughly as $1 / A$, where $A$ is proportional to $C_{2}^{3}$, or $r^{3} g^{3 / 2} \rho^{-1 / 2}$. In conclusion, $M_{\text {peak }}$ should vary roughly $a r^{-3}$, that is in proportion with the density of the ambient, $\rho_{\mathrm{a}}$.

The density of the ambient decreases by a factor of $10^{3}$ from animal swimmers to animal fliers. Figure 2 shows that the body size of the fastest animals decreases by the same factor from swimmers to fliers. This suggests that the clouds of data for swimmers and fliers would fill the same cloud if on the abscissa the body sizes (M) are multiplied by $\mathrm{r}^{3}$, with $\mathrm{r} \sim 10$ for fliers, $\mathrm{r} \sim 1$ for swimmers, and an in-between $\mathrm{r}$ value such as 3 for runners. 

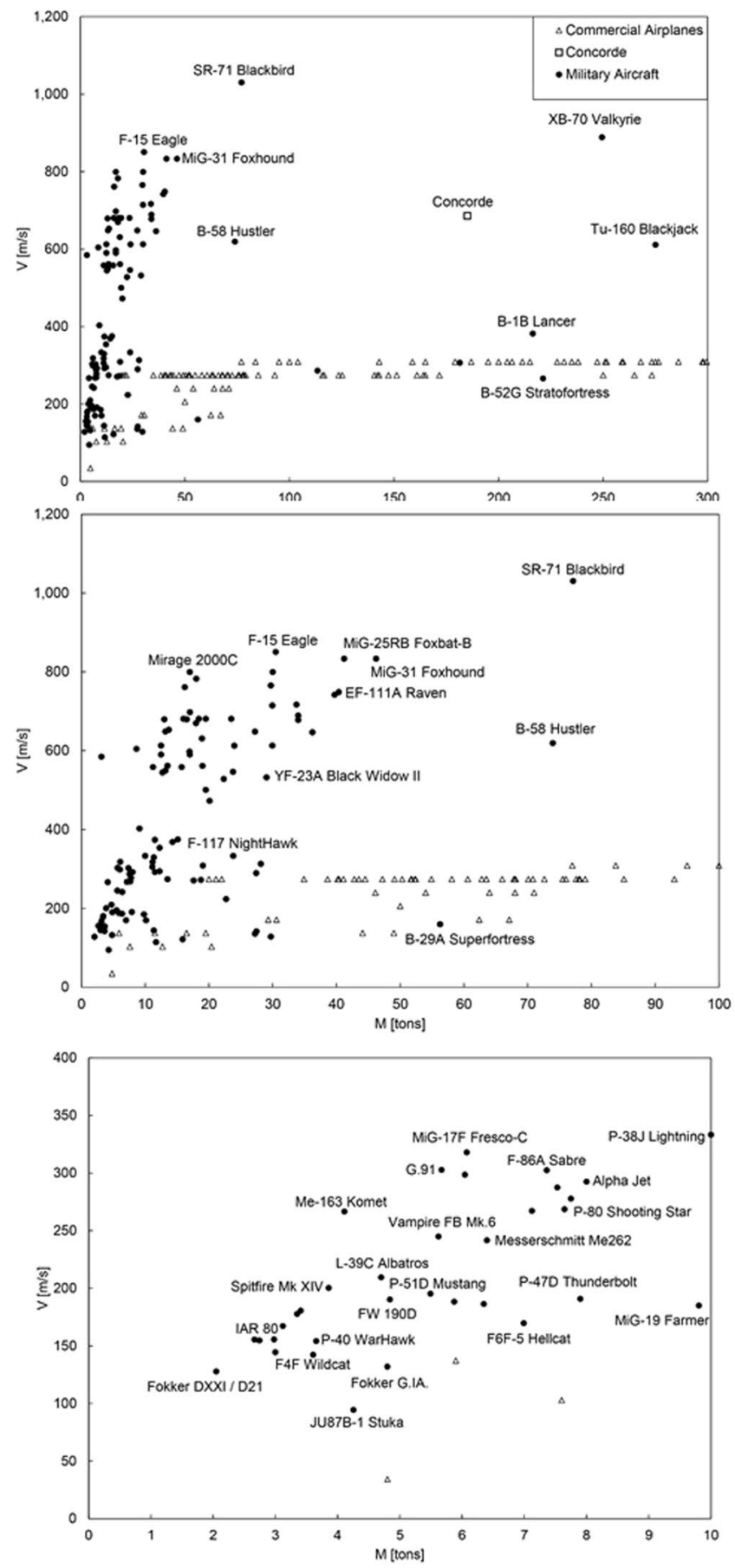

Figure 6. The evolution of speed in the design of military attack aircraft. The data are tabulated in Supplementary Material.

The theory advanced in this paper is less conclusive with regard to the effect of the ambient on the peak speeds of animals, fliers versus swimmers. According to Eq. (18), the peak speeds should vary as $M_{\text {peak }}^{1 / 6}$, which means that $\mathrm{V}_{\text {peak }}$ should vary roughly as $\mathrm{r}^{-1 / 2}$, or $\left(\rho / \rho_{\mathrm{a}}\right)^{-1 / 6}$. Taking $\mathrm{r} \approx 10$ for fliers and $\mathrm{r} \approx 1$ for swimmers, the prediction is that $\mathrm{V}_{\text {peak }}$ for fliers should be $1 / 3$ of the $\mathrm{V}_{\text {peak }}$ for swimmers. The opposite trend is exhibited by the data in Fig. 2.

\section{Economies of scale}

Bigger movers are more efficient movers. This aspect of the physics of powered locomotion is the basis of the present theory, starting with Eq. (2) with $\alpha<1$, and Fig. 3. This phenomenon is recognized more generally as economies of scale. Here we have the opportunity to show that the phenomenon is visible not only after plotting engine efficiencies versus size, as in Fig. 3, but also by looking at how airplanes are configured. 

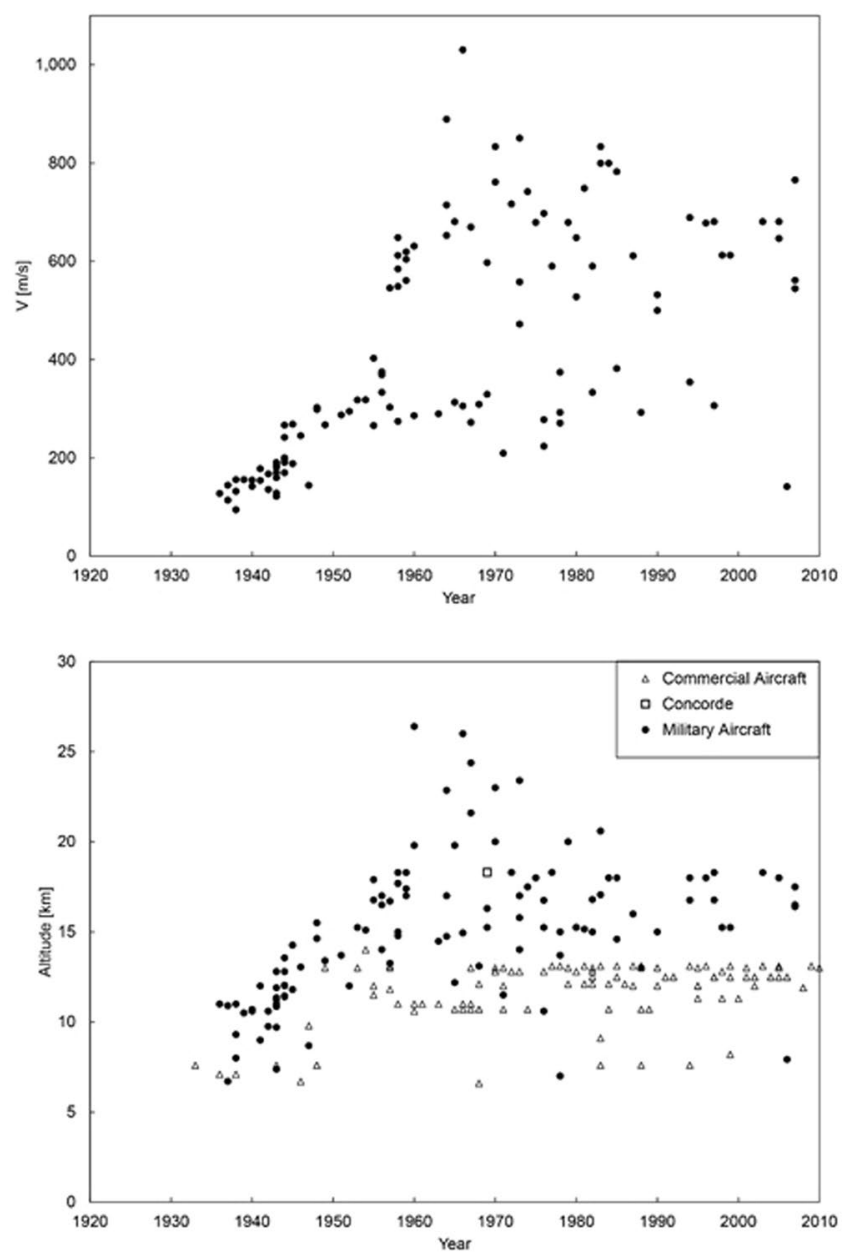

Figure 7. The timeline of the speed and altitude ceiling reached by military aircraft.

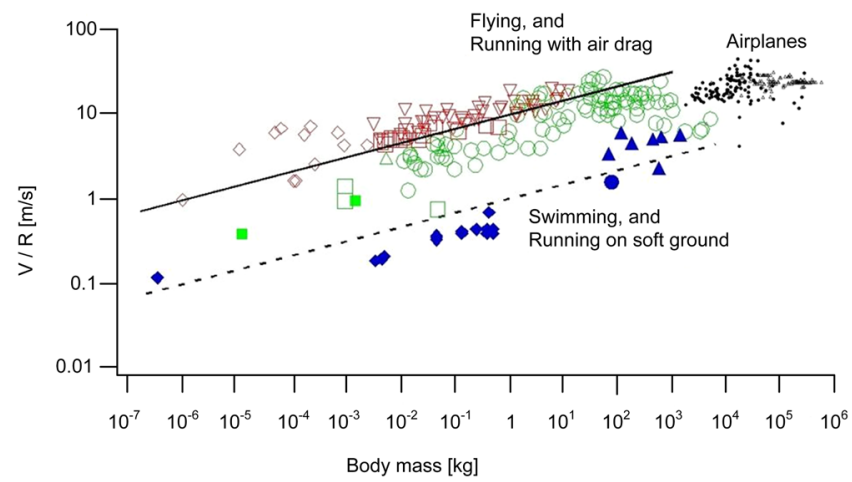

Figure 8. Revised version of Fig. 1, showing that the aircraft data line up with the animal fliers when the effect of lower air density and drag at higher altitudes is taken into account.

In Fig. 9 and Table 1 we used three airplane models to illustrate the evolution of the biggest transport aircraft that flies today. From the B52 to the B747 and the B777, the engine thrust (the force) and size (mass) increased by a factor of order 10. The number of engines decreased by a similar factor, from 8 to 4 , and finally 2 .

From the point of view of the whole vehicle, the evolution means something different. The data on the right side of Fig. 9 show the total thrust and total mass of the engines that drive the airplane. The data line up on the vertical, which means that the B52 from 1956 is driven by the same engine mass as the B747 of 1984 and the B777 of 2004. The difference and the message are on the vertical. From the 1950s to the 1980s, the total engine mass is essentially the same, the total thrust increased by an order of magnitude, and the size of the vehicle increased by a factor of the same order, from the B52 to the B747 and B777.

In sum, the evolution has been toward bigger force, bigger size, and more efficient engines, over time. 


\begin{tabular}{|l|l|l|l|}
\hline & B52-D & B747-400 & B777-300ER \\
\hline Year & 1956 & 1984 & 2004 \\
\hline Aircraft range [km] & 14,080 & 13,490 & 13,650 \\
\hline Aircraft fuel capacity [liters] & 181,610 & 216,840 & 181,283 \\
\hline Aircraft loaded mass [kg] & 120,000 & 396,890 & 351,533 \\
\hline Aircraft fuel efficiency [kg.km/1] & 9,303 & 24,694 & 26,469 \\
\hline Engine & Pratt \& Whitney J57-P-23 & GE CFM-80C2 & GE 90 115-B \\
\hline Number of engines & 8 & 4 & 2 \\
\hline Engine length [m] & 6.19 & 4.27 & 7.28 \\
\hline Engine fan diameter [m] & 0.99 & 2.69 & 3.3 \\
\hline Engine dry mass [kg] & 2347 & 4350 & 8762 \\
\hline Engine thrust [kN] & 52 & 260 & 513.9 \\
\hline Engine specific fuel consumption mg/(N.s) & 59 & 9.32 & 8.31 \\
\hline Engine fuel heating value [MJ/kg] & 43.15 & 44.65 & 44.65 \\
\hline
\end{tabular}

Table 1. Engine evolution in the three of the largest aircraft flying today ${ }^{14-19}$.

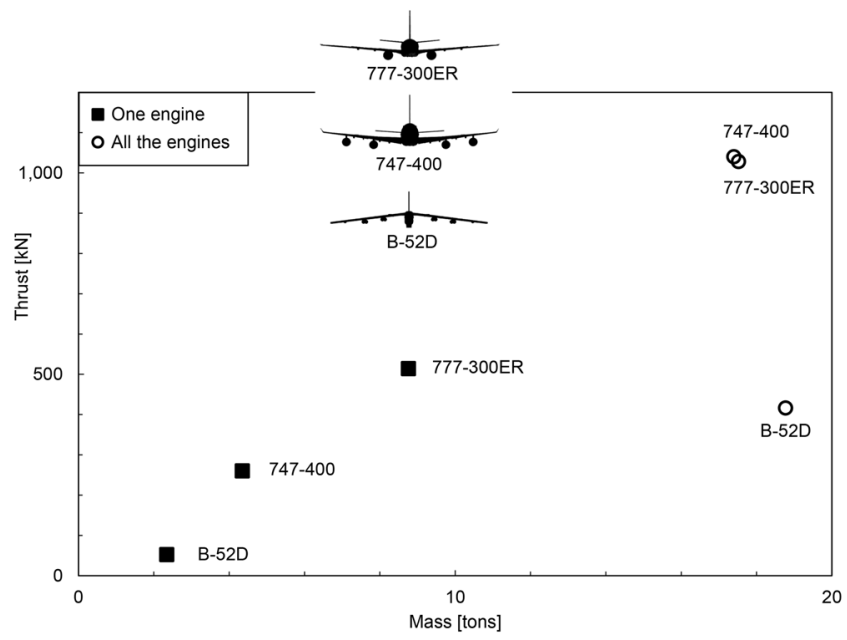

Figure 9. The evolution of the thrust and number of jet engines on three of the largest aircraft in service today (Table 1).

\section{Concluding remarks}

In this paper we relied on physics to show how the body size controls the maximum speed through the environment. The original challenge was to predict from theory that 'outliers' such as the cheetah must exist. The physical reason for outliers is that animals do not move the same way during their daily life. Grazing animals move constantly and eat constantly, while predators move and eat in spurts. In fact, predators spend most of their lives at rest, sleeping or watching the prey.

We showed that what accounts for the animal outlier (higher speed at smaller body mass) also accounts for the vehicle outlier. Military aircraft for chase, attack and reconnaissance are smaller and reach speeds higher than the biggest commercial aircraft. Yet, like the cheetah, the jet fighter spends most of its active life at rest, on the ground, out of sight.

The theoretical line pursued in this paper revealed two unexpected opportunities to complete the physics theory of animal and human flight. One was the deviation of the commercial aircraft data (upward, relative to animal data) in Fig. 1. The cause is the higher altitude of flying aircraft, where the air density is lower than close to the ground, and consequently the theoretical speeds must be higher. When the flying altitude effect is taken into account, the animal and aircraft data line up together (Fig. 8).

The second unexpected opportunity was to explain why the fastest animal fliers are $10^{3}$ times smaller than the fastest animal swimmers (Fig. 2). The explanation is in the theoretical formulas for maximum-speed locomotion. The body size for maximum speed depends on the density of the medium $\left(\rho_{a}\right)$ through which the animal is moving. The theoretical body size is approximately proportional to $\rho_{\mathrm{a}} / \rho$, and this ratio decreases by a factor of $10^{3}$ from the $\rho_{\mathrm{a}}$ of water to the $\rho_{\mathrm{a}}$ of air.

The practical usefulness of these theoretical advances is that they help us fast-forward the evolution of vehicle technology. This benefit was already demonstrated with the transition from the physics theory of all animal locomotion ${ }^{1,4}$, which revealed the main scaling laws of all moving animal design, to predicting the scaling laws of 


\begin{tabular}{|l|l|l|l|l|l|}
\hline & F-18A & $\mathbf{7 3 7 - 8 0 0}$ & $\mathbf{A 3 2 0 - 2 0 0}$ & A330-300 & B777-300ER \\
\hline Loaded mass $(\mathrm{kg})$ & 23,541 & 79,016 & 78,000 & 242,000 & 351,533 \\
\hline Max speed $(\mathrm{km} / \mathrm{hr})$ & 1,915 & 905 & 871 & 914 & 933 \\
\hline Cruise speed $(\mathrm{km} / \mathrm{hr})$ & 1,062 & 842 & 829 & 871 & 892 \\
\hline Range $(\mathrm{km})$ & 2,017 & 5,436 & 6,100 & 11,750 & 13,650 \\
\hline $\begin{array}{l}\text { Design flight service life } \\
\text { (in air time only) (hrs) }\end{array}$ & 10,000 & 60,000 & 60,000 & 100,000 & 125,000 \\
\hline Maximum hypothetical lifetime distance $\left(10^{6} \mathrm{~km}\right)$ & 10.6 & 50.5 & 49.7 & 87.1 & 115.0 \\
\hline Flight hours per year (hrs) & 330 & 3,200 & 3,200 & 4,380 & $5,219.5$ \\
\hline Expected total service life (in air time+ ground time) (hrs) & $265,454.5$ & 164,250 & 164,250 & 200,000 & $209,790.21$ \\
\hline $\begin{array}{l}\text { Average speed over } \\
\text { total aircraft life }(\mathrm{km} / \mathrm{hr})\end{array}$ & 40.01 & 307.58 & 302.83 & 435.5 & 531.48 \\
\hline
\end{tabular}

Table 2. Lifetime aircraft speed, averaged over flight time plus ground time $\mathrm{e}^{14,20-27}$.

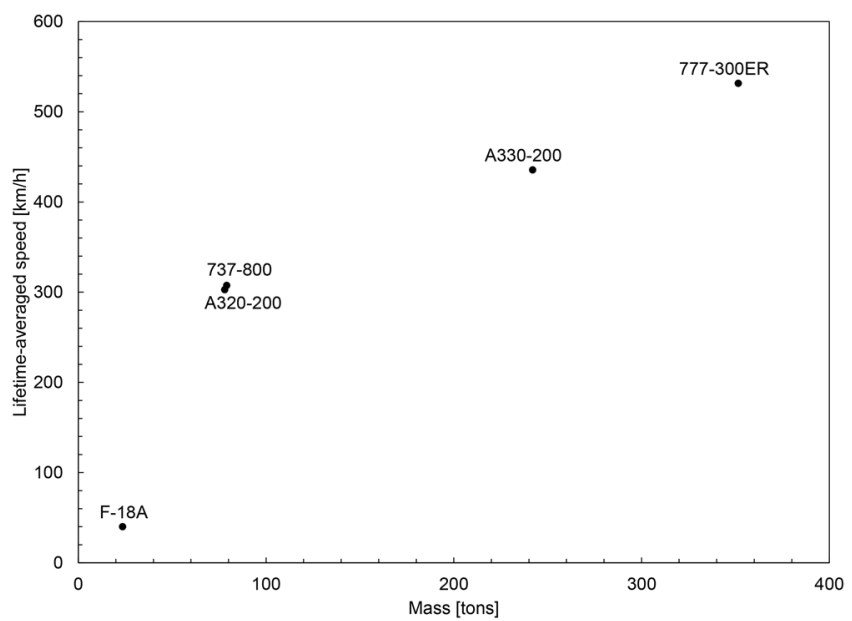

Figure 10. The lifetime-averaged speeds of current aircraft, showing the new 'outlier' position of the jet fighter (Table 2).

commercial airplanes ${ }^{6}$ and helicopters ${ }^{7}$, which have 'converged' naturally (from numerous disconnected designers and groups) during the documented lives of these technologies. It stands to reason that with the new theory and the morphological trends of modern aircraft reported in the present paper (e.g., sections 2-8 and Figs 5-10), designers, teams and industries will see more directly and more clearly the future of such vehicles. This predictive power of evolutionary design returns the favor to biology, for which it provides ample evidence of the natural phenomenon of evolution, which is observable in our lifetime ${ }^{13}$.

The speed 'outlier' phenomenon covered in this paper can be read from the much older perspective offered in Aesop's fable "The Tortoise and the Hare". What matters in the life of the mover is the movement (the territory covered, the speed averaged) over the lifetime. In Table 2 and Fig. 10 we brought together the lifetime speed and distance data of the work horses of commercial aviation and one jet fighter, the F-18A. This new presentation gives the word 'outlier' an entirely different meaning: the jet fighter is the outlier because during its lifetime it is slower than the bigger, the commercial aircraft. This new meaning is in fact the oldest, taught by Aesop.

\section{References}

1. Bejan, A. \& Marden, J. M. Unifying constructal theory for scale effects in running, swimming and flying. J. Exp. Biol. 209, 238-248 (2006).

2. Hirt, M. R., Jetz, W., Rall, B. C. \& Brose, U. A general scaling law reveals why the largest animals are not the fastest. Nat. Ecol. Evol. 1, 1116-1122 (2017).

3. Bejan, A. interviewed in S. Devos, Sa vitesse cache une théorie de la masse. Science \& Vie 75-77 (2017).

4. Bejan, A. Why the bigger live longer and travel farther: animals, vehicles, rivers and the winds. Sci. Rep. 2, 594 (2012).

5. Bejan, A., Almerbati, A. \& Lorente, S. Economies of scale: The physics basis. J. Appl. Phys. 121, 594, 044907 (2017).

6. Bejan, A., Charles, J. D. \& Lorente, S. The evolution of airplanes. J. Appl. Phys. 116, 044901 (2014).

7. Chen, R., Wen, C. Y., Lorente, S. \& Bejan, A. The evolution of helicopters. J. Appl. Phys. 120, 014901 (2016).

8. Meier, N. Jet Engine Specification Database. Available at: http://jet-engine.net/ (Accessed: 28th February 2018).

9. Vogt, W. P. \& Johnson, B. Dictionary of Statistics \& Methodology: A Nontechnical Guide for the Social Sciences. (SAGE Publications, 2011).

10. Fighter Planes. Fighter Planes Available at: https://www.fighter-planes.com/ (Accessed: 21st May 2018)

11. Chambers, M. C. et al. Analytical Fuselage and Wing Weight Estimation of Transport Aircraft. (1996).

12. U. S. Standard Atmosphere Supplements, 1966. 292 (NASA, 1966).

13. Bejan, A. The Physics of Life: The Evolution of Everything (St. Martin's Press, New York, 2016). 
14. 777-300ER. GE Aviation Available at: https://www.swiss.com/CMSContent/web/SiteCollectionDocuments/777/SWISS_Factsheet_ B777-300ER_EN.pdf (Accessed: 28th February 2018).

15. Boeing 747-400. Boeing Available at: http://www.boeing.com/resources/boeingdotcom/company/about_bca/startup/pdf/ historical/747-400-passenger.pdf (Accessed: 28th February 2018).

16. B-52 Stratofortress. U.S. Air Force Available at: http://www.af.mil/About-Us/Fact-Sheets/Display/Article/104465/b-52-stratofortress/ (Accessed: 28th February 2018).

17. Connors, J. \& Allen, N. The Engines of Pratt \& Whitney: A Technical History. (American Institute of Aeronautics and Astronautics, 2010).

18. The CF6 Engine. GE Aviation Available at: https://www.geaviation.com/commercial/engines/cf6-engine (Accessed: 28th February 2018).

19. The GE90 Engine. GE Aviation Available at: https://www.geaviation.com/commercial/engines/ge90-engine (Accessed: 28th February 2018).

20. F/A-18 Hornet strike fighter. The US Navy Fact FileAvailable at: http://www.navy.mil/navydata/fact_display.asp?cid=1100\&tid= 1200\&ct $=1$ (Accessed: 28th February 2018).

21. Boeing 737-800. Boeing Available at: http://www.airliners.net/aircraft-data/boeing-737-800900/96 (Accessed: 28th February 2018).

22. Airbus A320-200. GE Aviation Available at: http://www.airbus.com/aircraft/passenger-aircraft/a320-family/a320neo.html (Accessed: 28th February 2018).

23. Airbus A330-300. GE AviationAvailable at: http://www.airbus.com/aircraft/passenger-aircraft/a330-family/a330-300.html (Accessed: 28th February 2018).

24. Cooper, C. Bridging the Gap: Extending the Life of the F18 Hornet. (Master of Military Studies United States Marine Corps Command and Staff College Marine Corps University, 2011).

25. Aircraft and Related Datasets. MIT Global Airline Industry Program - Airline Data Project Available at: http://web.mit.edu/ airlinedata/www/Aircraft\&Related.html (Accessed: 21st May 2018).

26. Chang, F.-K. Structural Health Monitoring 2000. (CRC Press, 1999).

27. Jiang, H. Key Findings on Airplane Economic Life. Boeing White Pap. 9 (2013).

\section{Acknowledgements}

Prof. Bejan's work was supported by the US National Science Foundation. Umit Gunes' visit at Duke University was supported by the Scientific and Technological Research Council of Turkey (TÜBİTAK).

\section{Author Contributions}

A. Bejan and B. Sahin constructed the model. U. Gunes, and J. Charles performed the analysis numerically, and collected the new data for fast airplanes compiled in Supplementary Material. The text was written in multiple iterations by A. Bejan, B. Sahin, J. Charles and U. Gunes. The figures were made in multiple iterations by U. Gunes. and J. Charles.

\section{Additional Information}

Supplementary information accompanies this paper at https://doi.org/10.1038/s41598-018-30303-1.

Competing Interests: The authors declare no competing interests.

Publisher's note: Springer Nature remains neutral with regard to jurisdictional claims in published maps and institutional affiliations.

(c) (i) Open Access This article is licensed under a Creative Commons Attribution 4.0 International

License, which permits use, sharing, adaptation, distribution and reproduction in any medium or format, as long as you give appropriate credit to the original author(s) and the source, provide a link to the Creative Commons license, and indicate if changes were made. The images or other third party material in this article are included in the article's Creative Commons license, unless indicated otherwise in a credit line to the material. If material is not included in the article's Creative Commons license and your intended use is not permitted by statutory regulation or exceeds the permitted use, you will need to obtain permission directly from the copyright holder. To view a copy of this license, visit http://creativecommons.org/licenses/by/4.0/.

(c) The Author(s) 2018 